\title{
Mid- and long-term outcomes of endoscopic resection for submucosal esophageal cancer types pT1b-SM1 and pT1b-SM2
}

(이요 $\odot$

\author{
Authors \\ Institution \\ Department of Esophageal Surgery, Tokyo Medical and \\ Dental University, Tokyo, Japan \\ submitted 15.6.2018 \\ accepted after revision $\quad 27.12 .2018$ \\ Bibliography \\ DOI https://doi.org/10.1055/a-0838-5180 | \\ Endoscopy International Open 2019; 07: E733-E742 \\ (c) Georg Thieme Verlag KG Stuttgart · New York \\ eISSN 2196-9736 \\ Corresponding author \\ Kenro Kawada, MD, PhD, 1-5-45 Yushima, Bunkyo-Ku, \\ Tokyo, 113-8519, Japan \\ Fax: +81-3-3817-4126 \\ kawada.srg1@tmd.ac.jp
}

Andres Mora, Kenro Kawada, Yasuaki Nakajima, Takuya Okada, Yutaka Tokairin, Tatsuyuki Kawano

\section{ABSTRACT}

Background and study aims Endoscopic submucosal dissection (ESD) and endoscopic mucosal resection (EMR) are promising therapeutic options for early esophageal cancer (EC). The factors that can affect mid- and long-term survival in patients with submucosal EC (SM1 and SM2) have not been described in the literature. We aim to describe clinicopathological outcomes and factors that can affect the mid-and long-term survival in patients with resected submucosal tumors.

Patients and methods We performed a retrospective analysis of patients who underwent endoscopic resection (ER) for submucosal tumors over a 20 -year period. The final study population included 119 cases with 137 lesions. Information was collected according to the Japanese Classification of Esophageal Cancer 11-edition and factors affecting survival for 2 and 5 years after ER were analyzed.

Results EMR was performed in 99 cases (72.3\%), ESD in 38 cases $(27.7 \%)$. There were no significant complications. Two- and 5-year survival rates were $91 \%$ and $82 \%$, respectively. Mean age was 67.22 years ( \pm 9.49 years), mortality caused by EC occurred in 13 cases (11\%). Factors that had a significant impact on long-term survival were age $>65$ years $(P=0.0026)$, number of resected specimens $(P=$ 0.0031 ), presence of another progressive disease (not EC) $(P \leq 0.001)$, recurrence $(P=0.0002)$, and relation between histopathological positive vertical margin and recurrence $(P=0.0112)$.

Conclusions ER is viable treatment for esophageal submucosal cancer, selection between ESD/EMR can depend on tumor size and patient condition, and en bloc ER is the recommended technique for submucosal tumors. Long-term survival factors were identified.

\section{Introduction}

Esophageal cancer (EC) is one of the 10 most common newly diagnosed cancers in the world and the sixth most common cause of death from cancer [1]. In Japan, estimated incidence in 2009 was 17,492 for men and 3,295 for women, with a crude rate of 28.2 and 5.0 respectively; the incidence rate was $3.9 \%$ for men and $1 \%$ for women [2].

Endoscopic resection (ER) techniques, such as endoscopic mucosal resection (EMR) and endoscopic submucosa dissection (ESD), are established therapeutic modalities for EC and are associated with good outcomes [3]. Recent findings describe ESD as being more effective for resection of early EC than EMR, with better results with regard to en bloc resection and less recurrence. ESD has mainly become the standard technique in Japan and other Asian countries because it is associated with decreased local recurrence and improved survival [4]. However, we should not underestimate the potential of EMR in treatment of small and superficial tumors because it also has good results with en bloc resection and curative resection rates [5].

ER is suitable for superficial EC and choice of technique must be based on tumor size and thickness. Since 1989 there have been improvements in endoscopic diagnosis techniques and treatments for superficial EC, including new technologies and refined techniques, which have achieved good cure rates [6] Currently, lesions are removed under EMR with a snare or 
suctioned into a cap and snared only when tumors are small $(<2 \mathrm{~cm})$ and superficial mucosal. With an ESD procedure involving removal of the submucosa under the lesion with a specialized knife, larger and potentially deeper lesions can be treated. Depth of tumor invasion in endoscopic treatment, according to the Japanese Classification of Esophageal Cancer, 11th Edition, is described as the histologically deepest point of direct invasion of the primary tumor. In submucosal cancer, this point is the distance from the lamina muscularis mucosae to the deepest point of invasion. Therefore, submucosal cancer is subclassified as PT1b-SM1 (SM1; limited to within $200 \mu \mathrm{m}$ ) and PT1bSM2 (SM2; > $200 \mu \mathrm{m})$ [7].

Both EMR and ESD are noninvasive and less expensive treatments for EC that is limited to the mucosa without lymph node metastasis [8]; however, in patients with submucosal tumors, indications as a definitive treatment remain to be fully established.

Japanese guidelines for diagnosis and treatment of EC provide indications for ER: relative indications for MM or SM1 lesions that are not accompanied by clinical evidence or lymph node metastasis, and investigational indications for SM2 or deeper lesions targeted for local control, $50 \%$ of which are associated with metastasis [8]. In both types (SM1 and SM2), follow-up is mandatory, and additional treatment (radical surgery or adjuvant therapy) may be required, depending on the histopathological conditions of free border tumor [9].

In this study, we reviewed clinicopathological characteristics and factors that can affect mid- and long-term survival outcomes of patients who underwent under therapeutic treatment with EMR or ESD with SM1/SM2 EC tumors over a 20year period.

\section{Patients and methods}

The study protocol was approved by the Human Ethics Review Committee of Tokyo Medical and Dental University (No. M2017-332).

\section{Patients}

We performed a retrospective study of data that were prospectively collected in our institution. The dataset contained all information on patients with EC in whom ER was conducted from June 1995 to June 2015. From the 20 years of medical records, we included all patients with superficial clinical tumors who underwent ER for therapeutic purposes and a histopathologic diagnosis of T1b-SM1 and T1b-SM2, as assessed by the Japanese Guidelines for Diagnosis and Treatment of Carcinoma of the Esophagus by the Japan Esophageal Society. Clinicopathological characteristics were collected according to the Japanese Classification of Esophageal Cancer 11th edition [7, 10]. We excluded patients with T1a mucosa tumors, patients who received radiation or chemotherapy previously for the primary disease, and patients who had gastroesophageal junction tumors or advanced synchronous cancer of other organs. Also, three patients were excluded for travel abroad during the observation period. The final analysis included 137 lesions in 119 pa- tients diagnosed with submucosal tumor invasion (T1b-SM1 and T1b-SM2).

Preoperative evaluation of the tumor depended on the year in which the diagnosis was made and the hospital guidelines at the corresponding time. In all cases, patients underwent a physical examination, preoperative blood testing, imaging and endoscopic measurement. In most cases, barium X-ray and fullbody computed tomography were performed. Records from the surgery, endoscopy, and anesthesia departments were reviewed, as well as records from hospitalization and after patient discharge with continuous observation until attainment of tumor-free status, transfer to another institution, or death. All studied patients were observed for a minimum of 2 years (procedure in 2015) and for a maximum of 20 years (procedure in 1995).

\section{Procedure}

ER consisted of EMR and ESD. In our institution, the first endoscopic procedure was introduced in 1989 by professor Kawano T. and supported by professor Endo M. [11]. The first cases of EMR were performed using a transparent overtube with intraluminal negative pressure [12]. In 1992, the technique was improved using a cap-fitted panendoscope [13] and the technology for the method has been updated continuously. ESD was introduced in mid-2005, with numerous improvements up to the currenty used procedure. For this study, the first EMR was performed in 1996, and the first ESD in late 2005. Before the introduction of ESD, all endoscopic procedures for superficial clinical mucosa stages were completed by EMR without size distinction in 45 cases. Of them, only 17 cases were tumors $>2 \mathrm{~cm}$ and resected piecemeal. After 2005, the transition to ESD resulted in EMR procedures being omitted for superficial (cT1) and small tumors $(<2 \mathrm{~cm})$ that could be resected en bloc in elderly patients who had critical clinical conditions and were at high risk from general anesthesia ( $\mathbf{F i g . 1}$ ).

Information on the evolution of endoscopic procedures and techniques in our institution has been collected since the $1980 \mathrm{~s}$ and recently published [6].

ER is the indication for superficial tumors clinically diagnosed as T1a tumors (carcinoma in situ, lamina propia mucosae, and muscularis mucosae tumors) and T1b-SM1, and for T1b-SM2 tumors with relative indications and just for investigational cases, all based on the parameters of the Guidelines for Diagnosis and Treatment of Carcinoma of the Esophagus, edited by the Japan Esophageal Society [9]. ER is first-line treatment for superficial T1 and SM1 tumors (cN0) with curative intentions. Additional therapy may have been necessary, depending on histopathology of the specimen and the outcome of clinical. All patients who had lymphatic and/or venous positive invasion were candidates for radical surgery, with radiotherapy or chemotherapy reserved for patients with the same histopathological results but who had critical clinical conditions and for whom surgery represented a high risk. For clinical SM2 patients, we recommended radical esophagectomy or chemoradiation as first-line treatment. However, for patients whose status was compromised, we preferred ER ( $\triangleright$ Fig. 1). 


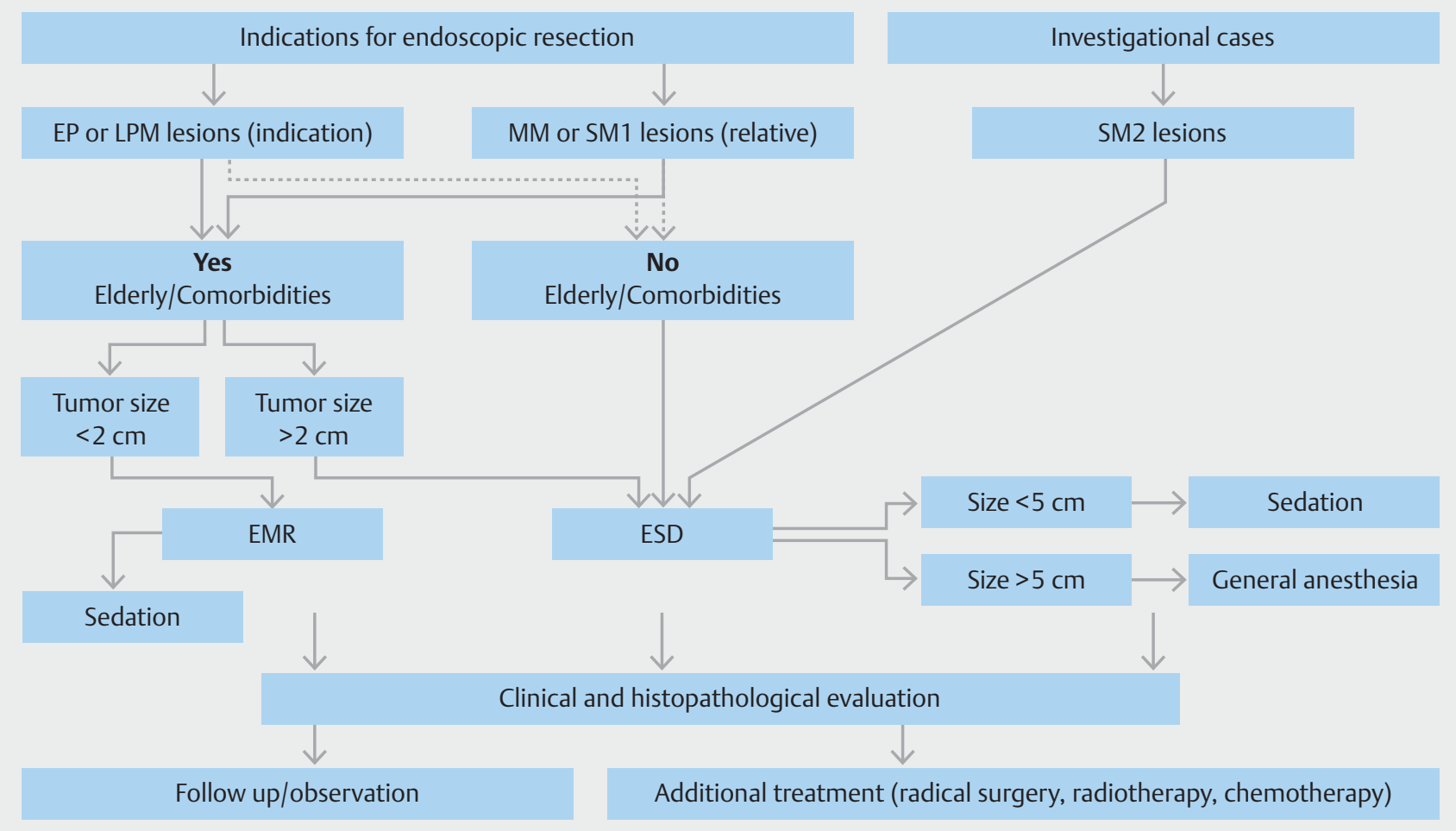

Fig. 1 Endoscopic procedure algorithm for superficial esophageal tumors. ER is the first line of treatment for clinical T1 and SM1 tumors, and SM2 tumors just for investigational cases.

Procedures were performed by qualified endoscopic surgeons; preoperative and postoperative care was handled by a first-class esophageal surgery team in a multidisciplinary system according to the guidelines of the Esophageal Surgery Department at Tokyo Medical and Dental University in Tokyo Japan.

\section{Statistical analysis}

Statistical analyses were performed using the JMP Base software program (version 13.0.0 2016, SAS Institute Inc). Survival was calculated using the Kaplan-Meier method and evaluated using the log-rank test; significance of differences regarding the characteristics of the patients and clinicopathological factors were determined using Cox proportional hazards regression model, chi-square test and Wilcoxon/Kruskal-Wallis test when appropriate. Statistical analyses were performed to identify any factors associated with 2- and 5-year survival after the procedure. The confidence interval was $95 \%$, and $P$ values $\leq$ 0.05 were considered to indicate statistical significance.

\section{Results}

\section{Characteristics of the study population}

Baseline characteristics are described in Table 1. Overall there were 119 cases with 137 lesions. Mean age \pm standard deviation was $67.22 \pm 9.49$ years, and $87.4 \%$ of the patients were male. Complications (including bleeding, stenosis, edema or subcutaneous emphysema) did not occur in any of the cases. A previous history of any cancer before the procedure was present in 21 cases (17.6\%), this included oropharyngeal cancer $(n=4,3.4 \%)$, gastric cancer $(n=4,3.4 \%)$, hypopharyngeal $(n=2,1.7 \%)$, lung $(n=2,1.7 \%)$, and colon cancer $(n=2,1.7 \%)$. Multiple primary cancers of the esophagus (metachronous) were present in 17 cases for a second primary (14.3\%), and one case for third primary $(0.8 \%)$, or a total 18 patients (15.1\%). Multi-organ primary early cancer (synchronous) was present in 18 cases (15.1\%). The most common types were hypopharyngeal cancer $(n=6,5 \%)$, gastric cancer $(n=6,5 \%)$ and oropharyngeal cancer $(n=3,2.5 \%)(\triangleright$ Table 1$)$.

\section{Macroscopic and histological findings}

EMR and ESD were performed for 99 (72.3\%) and 38 (27.7\%) lesions, respectively. The most common tumor location was the thoracic esophagus (86.8\%), specifically the middle thoracic esophagus (51.1\%). Macroscopic tumor type in most cases was 0 -Ilc (63.5\%), followed by 0 -I (10.2\%). Clinical diagnosis of tumor depth in a previous procedure was cT1a in 61 cases (44.5\%), cSM1 67 cases (48.9\%), and cSM2 9 cases (6.6\%). According to pathological tumor size, lesions $<2 \mathrm{~cm}$ occurred in 84 cases $(61.3 \%)$, and $>2 \mathrm{~cm}$ in 53 cases (38.7\%) with a total mean size of $2.24 \mathrm{~cm}( \pm 1.29 \mathrm{~cm})$. Finally, a positive vertical margin was present in 13 cases (9.5\%). En bloc resection and piecemeal resection were performed in $44.5 \%$ and $55.5 \%$ of all cases, respectively. Squamous cell carcinoma was the main histological type ( $n=134,97.8 \%$ ); the other three specimens were malignant melanoma, carcinosarcoma, and adenosquamous 
- Table 1 Baseline patient characteristics.

\begin{tabular}{|c|c|c|}
\hline & $\mathbf{n}$ & $\%(n / 119)$ \\
\hline Age mean $( \pm S D)$ & $67.22( \pm 9.49)$ & \\
\hline \multicolumn{3}{|l|}{ Gender } \\
\hline - Male & 104 & 87.4 \\
\hline - Female & 15 & 12.6 \\
\hline Total patients & 119 & 100.0 \\
\hline Total lesions & 137 & \\
\hline $\begin{array}{l}\text { - Multiple primary EC } \\
\text { (2nd primary) }\end{array}$ & 17 & 14.3 \\
\hline $\begin{array}{l}\text { Multiple primary EC } \\
\text { (3th primary) }\end{array}$ & 1 & 0.8 \\
\hline Previous history of cancer & 21 & 17.6 \\
\hline - Oropharyngeal cancer & 4 & 3.4 \\
\hline - Gastric cancer & 4 & 3.4 \\
\hline - Hypopharyngeal cancer & 2 & 1.7 \\
\hline - Lung cancer & 2 & 1.7 \\
\hline - Colon cancer & 2 & 1.7 \\
\hline - Other cancer & 7 & 5.9 \\
\hline Multiorgan primary cancer & 18 & 15.1 \\
\hline - Hypopharyngeal cancer & 6 & 5.0 \\
\hline - Gastric cancer & 6 & 5.0 \\
\hline - Oropharyngeal cancer & 3 & 2.5 \\
\hline - Others & 3 & 2.5 \\
\hline
\end{tabular}

SD, standard deviation; EC, esophageal cancer

carcinoma. Pathological diagnosis for depth of tumor invasion was T1b-SM 1 in $34.3 \%$ of the cases, and T1b-SM2 in $54.7 \%$. Lymphatic invasion and venous invasion were present in $37.2 \%$ and $43.1 \%$ of cases, respectively ( $\triangleright$ Table 2 ).

\section{Outcomes during follow-up}

Total follow-up was a mean of $73.4 \pm 51.36$ months and the maximum period of survival observed was 20.3 years. Followup 2 years after the procedure was reached in $100 \%$ of cases, and at 5 years in $68 \%$. Follow-up until final observation period was accomplished as follows: clinical observation in 76 cases $(63 \%)$ with a mean of $81.48 \pm 57.81$ months; discharged home without tumor in 39 cases (31\%) with a mean of $60.89 \pm 33.2$ months, and patients transferred to other institutions, four cases (3\%) with a mean $41.65 \pm 31.7$ months.

Incidence of multiorgan primary cancers (not EC) that developed during the follow-up period was $15 \%$ and the most common types were hypopharyngeal $(n=6,5 \%)$ and gastric cancer ( $n=6,5 \%)$, followed by oropharyngeal cancer $(n=3,3 \%)$. In three cases during the observation period, patients developed a second primary tumor eligible for EC after ER that was mucosal (3\%).

Additional treatment was offered, depending on results of clinical and histopathological evaluation, to all patients who had lymphatic and/or venous positive invasion, and/or non-tumor-free borders on the pathological specimen according to the guideline [9]. In our study, a total of 34 patients (29\%) received additional treatment; these consisted of radical esophagectomy $(n=23 ; 19 \%)$ and adjuvant therapy (CT-CRT) $(n=11$; $9 \%)$. The decision to treat was made on a case-by-case basis, depending on mainly histopathological findings. For patients SM1 without lymphatic or venous invasion, we did not recommend additional therapy, but if lymphovascular involvement was documented, we recommended additional treatment, taking into consideration a patient's status.

In 40 patients (34\%), progressive diseases (other than EC) developed during the observation period which were classified as conditions that can affect the quality of life. This included other types of progressive cancer such as second primary tumors ( $n=24 ; 20 \%)$, cardiorespiratory diseases $(n=8 ; 7 \%)$, and other conditions, such as renal, neurological or systemic disorders $(n=8 ; 7 \%)$. Overall during the observation period, 30 patients $(25 \%)$ died. Causes of death included EC ( $n=13 ; 11 \%)$; other types of progressive cancer $(n=7 ; 6 \%)$; and other progressive disorders $(n=10 ; 8 \%)$ ( Table 3$)$.

\section{Recurrence}

Characteristics of EC recurrence after ESD or EMR for submucosal tumors are shown in $>$ Table 4 . Recurrence occurred in 23 patients $(19 \%)$, mean tumor-free period after the procedure was $34.8( \pm 36.25)$ months, and survival time after a diagnosis of recurrence was a mean of $29.4( \pm 30.7)$ months. Overall in 23 patients who developed recurrent disease, EMR was performed in 16 cases (70\%), and ESD in 7 (30\%). Local and distant recurrence occurred in $15(65 \%)$ and 8 (33\%) cases respectively. Recurrence developed after SM1 procedures in 6 cases $(26 \%)$ and after SM2 diagnosis in 17 cases (74\%). In 6 cases (26\%), recurrence occurred in patients with positive vertical margin. Vascular and lymphatic invasion was present in 18 patients (78\%). Additional treatment was performed in 19 patients (83\%), nine cases with adjuvant therapy and 10 cases with radical and palliative surgery. Mortality occurred in 15 patients with recurrence $(65 \%)$, and cause of death was EC $(n=13)$; another type of cancer $(n=1)$, and other progressive disease $(n=1)$. According to the analysis, risk factors of recurrence affecting long-term survival in 5 years that rose to statistical significance were age $>65$ years $(P=0.0282)$, male gender $(0.00152)$, and positive vertical margin in the histopathological specimen (0.0311) ( $\triangleright$ Table 4).

\section{Survival period and factors affecting survival}

During the study period, 2- and 5-year survival rates as determined by a Kaplan-Meier analysis were $90 \%$ and $79 \%$, respectively ( $>$ Fig.2). Clinicopathological factors with the potential to affect survival were analyzed at 2 and 5 years after the endoscopic procedure. All previously reported factors were analyzed 
- Table 2 Macroscopic and histological findings in 137 lesions.

\begin{tabular}{|c|c|c|c|c|c|c|}
\hline & Primary Lesion & $\%$ & 2nd Primary & $\%$ & 3 rd Primary & $\%$ \\
\hline Total patients & 119 & 86.9 & 17 & 12.4 & 1 & 0.7 \\
\hline \multicolumn{7}{|l|}{ Primary treatment } \\
\hline . EMR & 87 & 63.5 & 12 & 8.8 & & \\
\hline - ESD & 32 & 23.4 & 5 & 3.6 & 1 & 0.7 \\
\hline \multicolumn{7}{|l|}{ Tumor Location } \\
\hline - Cervical esophagus & 13 & 9.5 & 0 & 0.0 & & \\
\hline - Upper thoracic esophagus & 18 & 13.1 & 1 & 0.7 & 1 & 0.7 \\
\hline - Middle thoracic esophagus & 59 & 43.1 & 11 & 8.0 & & \\
\hline - Lower thoracic esophagus & 26 & 19.0 & 3 & 2.2 & & \\
\hline - Abdominal esophagus & 3 & 2.2 & 2 & 1.5 & & \\
\hline \multicolumn{7}{|l|}{ Macroscopic tumor type } \\
\hline . $0-1$ & 14 & 10.2 & 2 & 1.5 & & \\
\hline " 0-II 0-IIa & 6 & 4.4 & 2 & 1.5 & 1 & 0.7 \\
\hline$-0-I I b$ & 5 & 3.6 & 5 & 3.6 & & \\
\hline$-0-I I c$ & 87 & 63.5 & 8 & 5.8 & & \\
\hline . $0-I I I$ & 7 & 5.1 & 0 & 0.0 & & \\
\hline \multicolumn{7}{|l|}{ Clinical diagnosis } \\
\hline - cT1a & 48 & 35.0 & 12 & 8.8 & 1 & 0.7 \\
\hline - cT1b SM1 & 62 & 45.3 & 5 & 3.6 & & \\
\hline - cT1b SM2 & 9 & 6.6 & 0 & 0.0 & & \\
\hline \multicolumn{7}{|l|}{ Number of specimens } \\
\hline - En bloc resection & 49 & 35.8 & 11 & 8.0 & 1 & 0.7 \\
\hline - Piecemeal resection & 70 & 51.1 & 6 & 4.4 & & \\
\hline Pathological vertical size & & & & 0.0 & & \\
\hline . $<2 \mathrm{~cm}$ & 72 & 52.6 & 11 & 8.0 & 1 & 0.7 \\
\hline . $>2 \mathrm{~cm}$ & 47 & 34.3 & 6 & 4.4 & & \\
\hline - Size Mean $( \pm S D)(m m)$ & $20( \pm 12.09)$ & & $32.8( \pm 18.98)$ & & $6( \pm 0)$ & \\
\hline Positive vertical margin & 13 & 9.5 & 0 & & 0 & \\
\hline \multicolumn{7}{|l|}{ Histological type } \\
\hline - Squamous cell carcinoma & 116 & 84.7 & 17 & 12.4 & 1 & 0.7 \\
\hline - Others & 3 & 2.2 & 0 & 0.0 & & \\
\hline Depth of tumor invasion & & 0.0 & & 0.0 & & \\
\hline - pT1b-SM 1 & 47 & 34.3 & 9 & 6.6 & & \\
\hline " pT1b-SM 2 & 75 & 54.7 & 8 & 5.8 & 1 & 0.7 \\
\hline Lymphatic invasion ly(+) & 51 & 37.2 & 5 & 3.6 & & \\
\hline Venous invasion $\mathrm{v}(+)$ & 59 & 43.1 & 7 & 5.1 & & \\
\hline
\end{tabular}




\begin{tabular}{|c|c|c|}
\hline & $\mathbf{n}$ & $\%(n / 119)$ \\
\hline $\begin{array}{l}\text { Follow-up period (mean }[ \pm \mathrm{SD}] \\
\text { months) }\end{array}$ & $73.40( \pm 51.36)$ & \\
\hline 2-year follow patients & 119 & 100 \\
\hline 5-year follow patients & 81 & 68 \\
\hline $\begin{array}{l}\text { 2nd primary tumor (mucosal } \\
\text { EC-observation period } 2-5 \mathrm{yr} \text { ) }\end{array}$ & 3 & 3 \\
\hline \multicolumn{3}{|c|}{ Other progressive diseases (Not EC) } \\
\hline - Second primary tumors & 24 & 20 \\
\hline - Gastric cancer & 8 & 7 \\
\hline - Colon cancer & 3 & 3 \\
\hline - Hypopharyngeal cancer & 3 & 3 \\
\hline - Oropharyngeal cancer & 2 & 2 \\
\hline - Lung cancer & 2 & 2 \\
\hline - Other cancer & 6 & 5 \\
\hline - Cardiorespiratory & 8 & 7 \\
\hline - Neurological & 4 & 3 \\
\hline - Renal & 2 & 2 \\
\hline - Others & 2 & 2 \\
\hline \multicolumn{3}{|l|}{ Additional treatment } \\
\hline - Adjuvant therapy (CT-CRT) & 11 & 9 \\
\hline - Surgery (3FLD) & 23 & 19 \\
\hline $\begin{array}{l}\text { Mortality overall observation } \\
\text { period }\end{array}$ & 30 & 25 \\
\hline Cause of death & & 0 \\
\hline - Esophageal cancer & 13 & 11 \\
\hline - Other cancer & 7 & 6 \\
\hline - Other progressive disease & 10 & 8 \\
\hline
\end{tabular}

EC, esophageal cancer; CT, chemotherapy; CRT, chemoradiotherapy; 3FLD, esophagectomy with three-field lymph node dissection

including vertical margin on the histopathological record positive vs negative and depth of tumor and recurrence. Overall analysis for 2-year survival after ER should that other progressive disease (not EC) was a significant factor $(P=0.0377)$. Also, in the cross-section of variables, the relationship between the positive margin in the sample of histopathology and patients with recurrence gave a significant statistical value for lower survival rate $(P=0.0271)$. For 5 -year survival, significant factors were: age (cutoff 65 years; $P=0.0026$ ), number of resected specimens (piecemeal resection vs en bloc; $P=0.0031$ ), other progressive disease (not EC) $(P \leq 0.001)$, and recurrent EC $(P=$ $0.0002)$. Finally, in the cross-section of variables, the relationship between patients who had recurrence with positive vertical margin in the histopathology specimen was associated with a statistical significantly lower survival rate $(P=0.0112)(\triangleright$ Table 5).

\section{Discussion}

In this study, we confirmed the effectiveness of endoscopic procedures such as ESD or EMR for superficial EC. Both techniques have specific recommendations, especially EMR only in selected cases. Factors that affected long-term survival included age ( $>65$ years), number of resected specimens (piecemeal resection vs. en bloc), other progressive disease, recurrent EC and the relationship between the positive vertical margin in the pathological specimen and recurrence.

Advanced age was shown to be significantly associated with mortality in EC. Even after radical treatment, patients over age 65 have more risk with any type of medical procedure [14]. In elderly patients with significant comorbidities, high risk of general anesthesia, superficial tumor and small tumor size $(<2 \mathrm{~cm})$, we prefer to perform EMR, mainly because it can be performed under sedation. In all other cases, ESD is the preferred technique for submucosal esophageal tumors ( $\mathbf{F i g} \mathbf{1}$ ).

Several reports showed that ESD is more effective for EC, with higher en bloc resection rate and a lower incidence of local recurrence in comparison to EMR [4,5], The effectiveness of resection by ESD and its en bloc removal has been described in multiple reports, while EMR may remove the tumor in more than one piece depending on the size and thickness. In our study, we confirmed that en bloc resection was significantly associated with long-term survival in comparison to piecemeal resection ( $\bullet$ Table 5 ). Also, we concluded that ESD is a superior technique in terms of the number of resected specimen purposes for en bloc resection, leaving EMR only useful for small and superficial tumors $(<2 \mathrm{~cm})$ in elderly patients with significant comorbidities, for whom receiving general anesthesia would be associated with a very high risk of death. Only in these cases can we achieve en bloc resection ( $\bullet$ Fig. $\mathbf{1}$ ).

Other progressive diseases - including different types of cancer and other diseases - affected mid- and long-term survival. Overall during the observation period, patients were diagnosed and treated for different types of cancer or/and other significant diseases. We believe this is indicative of the relationship between advanced age and multiple comorbidities. Overall, $35 \%$ of the patients developed another significant progressive disease that affected their quality of life during long-term follow-up ( $\triangleright$ Table 3 ).

Recurrence is another factor that affects survival. In our study, $19 \%$ of patients developed recurrent disease during the observation period. In recurrent cases, mortality occurred in 15 patients (65\%), with a mean survival period after diagnosis of recurrence of 29.4 months ( \pm 30.7 ); $70 \%$ of the recurrent cases occurred after EMR, and the histological depth was SM2 in 74\% of cases. However, no significant differences were observed between ESD and EMR, or SM1 and SM2 $(P>0.05)$.

The significantly higher rate of recurrence after EMR was due to the higher rate of piecemeal resection. We must consider that the initial ER techniques were performed until 2005 with EMR, usually in piecemeal resections with high risk of positive 
- Table 4 Recurrence of submucosal EC after ESD or EMR.

\begin{tabular}{|c|c|c|}
\hline & $\mathbf{n}$ & $\%(n / 119)$ \\
\hline \multirow[t]{2}{*}{ Patients with recurrence } & 23 & 19 \\
\hline & $\mathbf{n}$ & $\%(n / 23)$ \\
\hline Local recurrence & 15 & 65 \\
\hline Distant recurrence & 8 & 35 \\
\hline Recurrence in SM1 & 6 & 26 \\
\hline Recurrence in SM2 & 17 & 74 \\
\hline Recurrence in ESD & 7 & 30 \\
\hline Recurrence in EMR & 16 & 70 \\
\hline Recurrence in Positive Vertical Margin & 6 & 26 \\
\hline Vascular invasion ly $(+)+v(+)$ & 18 & 78 \\
\hline Tumor-free period (mean [ $\pm S D]$ months) & $34.8( \pm 36.25)$ & \\
\hline Survival after recurrence (mean $[ \pm S D]$ months) & $29.4( \pm 30.7)$ & \\
\hline \multicolumn{3}{|l|}{ Additional treatment } \\
\hline - Adjuvant therapy (CT-CRT) & 9 & 39 \\
\hline - Surgery (3FLD) & 10 & 43 \\
\hline Mortality after recurrence & 15 & 65 \\
\hline \multicolumn{3}{|l|}{ Cause of death } \\
\hline - Esophageal cancer & 13 & 57 \\
\hline - Other cancer & 1 & 4 \\
\hline - Other disease & 1 & 4 \\
\hline
\end{tabular}

\section{Risk Factor for Recurrence in long-term observation after ESD and EMR}

\begin{tabular}{|c|c|c|}
\hline & \multicolumn{2}{|l|}{5 -year } \\
\hline & $\mathrm{HR}(95 \% \mathrm{Cl})$ & $P$ value \\
\hline \multicolumn{3}{|l|}{ Age } \\
\hline . $<65$ & $1(-)$ & - \\
\hline . $>65$ yrs & $6.12(1.17-112.33)$ & $0.0282^{1}$ \\
\hline \multicolumn{3}{|l|}{ Gender } \\
\hline - Female & $1(-)$ & - \\
\hline - Male & $4,93(1.60-1.60)$ & $0.0152^{1}$ \\
\hline \multicolumn{3}{|c|}{ Positive vertical margin } \\
\hline - Absent & $1(-)$ & - \\
\hline - Present & $3.94(1.14-12.68)$ & 0.0311 \\
\hline \multicolumn{3}{|c|}{ Number of specimens } \\
\hline - En bloc & $1(-)$ & - \\
\hline - Piecemeal & $1.00(0.32-3.42)$ & 0.9875 \\
\hline \multicolumn{3}{|c|}{ Other progressive disease } \\
\hline - Absent & $1(-)$ & - \\
\hline - Present & $1.81(0.56-6.83)$ & 0.3202 \\
\hline
\end{tabular}




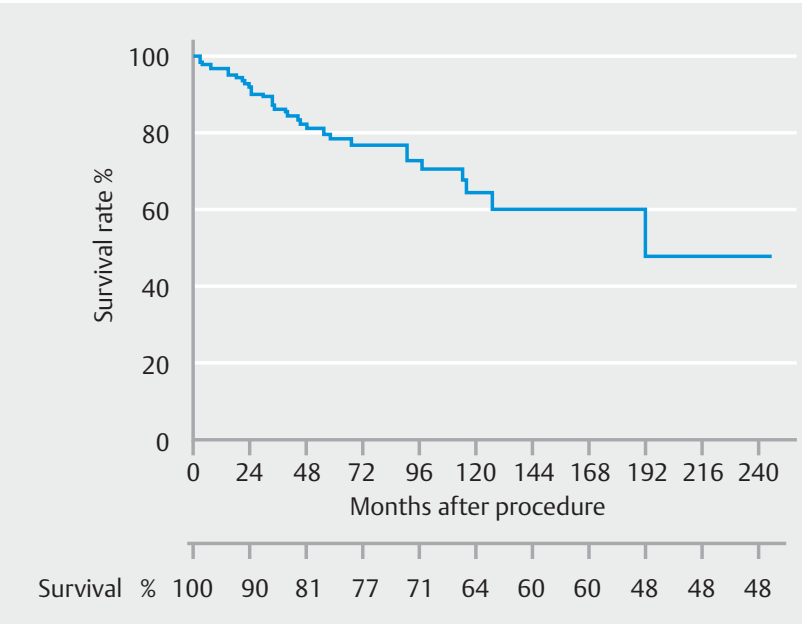

- Fig. 2 Survival of patients with submucosal tumors after ESD or EMR. Mean follow-up period was $73.4( \pm 51.36)$ months. The 2- and 5 -year survival rates were $90 \%$ and $79 \%$, respectively.

vertical margin. However, we believe that mortality is reduced with local control of recurrence and additional treatment in such cases. The relation between positive vertical margin in a specimen and recurrence was significant in 2- and 5-year follow-up. Given this antecedent, we concluded that the specimen must be resected en bloc with free tumor margin to avoid a positive vertical margin and its recurrence. In this study we have shown that cases with positive vertical margins are closely related to cases with recurrence, and their relationship statistically affected long-term survival ( $\downarrow$ Table 5 ). For this reason, we concluded that additional treatment is necessary for cases with lymphovascular involvement and/or positive margin in the histopathology specimen to decrease risk of recurrence and improve survival. That has already been described previously by other authors for curability criteria $[9,15]$. In our cases, the first line for additional therapy was surgery (3FLD), and chemotherapy for patients who were not eligible for surgical treatment, to avoid recurrence and complications as described in past studies of post-recurrence cases [16]. In our patients with tumor-free margins, we practice periodic observation for at least 5 years to provide early control of recurrence. In cases of any local or distal recurrence, we prefer surgery (3FLD) as first line because it produces good results, and chemotherapy for patients who cannot receive surgical treatment or who have distant metastasis $[6,14]$.

Risk factors for recurrence significantly associated with prognosis in our study were age (> 65 years), male gender, and positive vertical margin. The last one is the most critical factor in recurrence and affects survival in the long term after the procedure. Positive vertical margin also was identified in other studies by our department in addition to the longest diameter of the largest recurrent tumor, invasion into adjacent structures and speed of growth of the representative recurrent tumors $[16,17]$.

- Table 5 Factors affecting 2 - and 5-year survival after ESD or EMR in patients with submucosal tumors.

\begin{tabular}{|c|c|c|c|c|}
\hline & \multicolumn{2}{|l|}{ 2-year } & \multicolumn{2}{|l|}{ 5-year } \\
\hline & $\mathrm{HR}(95 \% \mathrm{Cl})$ & $P$ value & HR $(95 \% \mathrm{Cl})$ & $P$ value \\
\hline \multicolumn{5}{|l|}{ Age, years } \\
\hline . $<65$ & $1(-)$ & - & $1(-)$ & - \\
\hline . >65 years & $0.32(0.05-1.27)$ & 0.1140 & $0.20(0.04-0.60)$ & $0.0026^{1}$ \\
\hline \multicolumn{5}{|c|}{ Number of specimens } \\
\hline - En bloc & $1(-)$ & - & $1(-)$ & - \\
\hline - Piecemeal & $1.62(0.18-2.17)$ & 0.4462 & $0.46(0.28-0.77)$ & $0.0031^{1}$ \\
\hline \multicolumn{5}{|c|}{ Other progressive disease } \\
\hline - Absent & $1(-)$ & - & $1(-)$ & - \\
\hline - Present & $0.26(0.06-0.87)$ & $0.0377^{1}$ & $0.15(0.05-0.38)$ & $<0.001^{1}$ \\
\hline \multicolumn{5}{|l|}{ Recurrence } \\
\hline - Absent & $1(-)$ & - & $1(-)$ & - \\
\hline - Present & $0.40(0.12-1.42)$ & 0.1518 & $0.19(0.08-0.46)$ & $0.0002^{1}$ \\
\hline \multicolumn{5}{|c|}{ Recurrence with positive VM } \\
\hline - Absent & $1(-)$ & - & $1(-)$ & - \\
\hline - Present & $0.21(0.06-0.83)$ & $0.0271^{1}$ & $0.13(0.02-0.61)$ & $0.0112^{1}$ \\
\hline
\end{tabular}


In the current study, no associations were observed between any type of vascular invasion, SM1, or SM2 and mid- or longterm survival. However, lymphatic invasion and venous invasion were detected in $54 \%$ and $58 \%$ of recurrent cases, respectively. As is indicated under the guidelines and a statement with which we agree, additional treatment is strongly recommended for patients with any lymphovascular invasion, preferably radical surgery, if possible $[9,17]$.

Some studies have reported that adverse events (AE) occur in up to $20 \%$ of SM1 cases after ESD [15], with perforation occurring in a small number of cases, whereas other authors have described good outcomes in patients who underwent ER for superficial esophageal carcinoma [18]. ER is defined as a safe procedure, even in elderly patients with the proper technique [19]. No AEs were detected in our patients with either technique even in SM2 cases. We concluded and agree that ER is a safe and effective treatment for superficial esophageal carcinoma in elderly and non-elderly patients.

Esophageal cancer is often associated with other multiorgan primary cancers. Supporting the concept of field cancerization, synchronous multiple primary cancers may be an independent predictor of survival, and some reports have described simultaneous tumors [20]. In our study, $15 \%$ of cases had multiorgan primary cancers, and another $15 \%$ had metachronous tumors, with one patient having a third primary tumor at time of diagnosis. After the procedure in the follow-up period, we found that another $15 \%$ of patients had multiorgan primary cancer (not EC) and $3 \%$ had a second primary EC. Neither of these scenarios had a significant impact on long-term survival in our study. However, we support the field cancerization theory, which is consideration for complete screening of the digestive tract and promotion of early detection of second primary tumors.

The current study is associated with some limitations, including the relatively small number of cases, the fact that it was performed in a single institution, and its retrospective design, each of which can limit accuracy of the results. Furthermore, preoperative management, diagnostic methods, and postoperative care were heterogeneous. Also, the techniques and improvement in technology differed over the study period. Adaptation of the concepts for and consensus about treatment changed over the course of the 20 -year study period. These differences had the potential to influence the outcomes.

\section{Conclusion}

Based on the current study of endoscopically treated submucosal (SM1 and SM2) EC tumors, we conclude that endoscopic resection is a suitable option for submucosal tumors with good survival rate and long-term outcomes. For SM1 tumors, ER is the standard treatment. For SM2 tumors, treatment is controversial. In our study, we showed good survival results over the long term in SM2 cases without lymphatic or vascular involvement, free vertical margin and en bloc resection. Additional treatment is necessary for all SM tumors with lymphovascular involvement or positive vertical margins to avoid recurrence. Long-term follow-up is essential for identifying recurrence
(19\% incidence in our cohort) as it is associated with a high rate of mortality (65\%). An ER procedure should be selected based on the endoscopist's experience, with ESD as the preferred choice, and EMR an option for elderly patients with significant comorbidities, tumors less than $2 \mathrm{~cm}$ and for whom general anesthetic presents a high risk.

\section{Competing interests}

None

References

[1] Ferlay J, Soerjomataram I, Dikshit R et al. Cancer incidence and mortality worldwide: sources, methods and major patterns in GLOBOCAN 2012. Int J Cancer 2015; 136: E359-386

[2] Hori M, Matsuda T, Shibata A et al. Cancer incidence and incidence rates in Japan in 2009: a study of 32 population-based cancer registries for the Monitoring of Cancer Incidence in Japan (MCIJ) project. Jpn J Clin Oncol 2015; 45: 884-891

[3] Rizvi QU, Balachandran A, Koay D et al. Endoscopic Management of Early Esophagogastric Cancer. Surg Oncol Clin N Am 2017; 26: 179 191

[4] Nishizawa T, Yahagi N. Endoscopic mucosal resection and endoscopic submucosal dissection: technique and new directions. Curr Opin Gastroenterol 2017; 33: 315-319

[5] Ishihara R, lishi H, Uedo $\mathrm{N}$ et al. Comparison of EMR and endoscopic submucosal dissection for en bloc resection of early esophageal cancers in Japan. Gastrointest Endosc 2008; 68: 1066 - 1072

[6] Kawano T, Kamata K, Fujiwara N et al. History of endoscopic diagnosis and treatment of superficial esophageal cancer. Gastrointest Endosc 2018; 30: 129-136

[7] Japan Esophageal S. Japanese Classification of Esophageal Cancer, 11th Edition: part I. Esophagus 2017; 14: 1-36

[8] Committee AT, Maple JT, Abu Dayyeh BK et al. Endoscopic submucosal dissection. Gastrointest Endosc 2015; 81: 1311 - 1325

[9] Kuwano H, Nishimura Y, Oyama T et al. Guidelines for diagnosis and treatment of carcinoma of the esophagus April 2012 edited by the Japan Esophageal Society. Esophagus 2015; 12: 1-30

[10] Japan Esophageal S. Japanese Classification of Esophageal Cancer, 11th Edition: part II and III. Esophagus 2017; 14: 37-65

[11] Kawano T, Endo M. [Diagnosis and treatment of early esophageal carcinoma]. Nihon Rinsho 1989; 47: 1102-1106

[12] Kawano T, Miyake S, Yasuno M et al. A New technique for endoscopic esophageal mucosectomy using a transparent overtube with intraluminal negative pressure (np-EEM). Digest Endosc 1991; 3: 159- 168

[13] Inoue $\mathrm{H}$, Endo $M$, Takeshita $\mathrm{K}$ et al. A new simplified technique of endoscopic esophageal mucosal resection using a cap-fitted panendoscope (EMRC). Surg Endosc 1992; 6: 264-265

[14] Mora A, Nakajima Y, Okada T et al. Comparative study of predictive mortality scores in esophagectomy with three-field lymph node dissection in patients with esophageal cancer. Dig Surg 2019; 36: 67 - 75

[15] Qi ZP, Chen T, Li B et al. Endoscopic submucosal dissection for early esophageal cancer in elderly patients with relative indications for endoscopic treatment. Endoscopy 2018; 50: 839-845

[16] Nakajima Y, Kawada K, Tokairin Y et al. Prognostic factors for post-recurrence survival in patients with thoracic esophageal squamous cell carcinoma after curative resection. Dig Surg 2016; 33: 136-145 
[17] Kawano T, Nakajima Y, Suzuki T et al. [Esophageal carcinoma - from the viewpoint of surgery]. Gan To Kagaku Ryoho 2007; 34: 824-830

[18] Lee HJ, Lee H, Park JC et al. Treatment strategy after endoscopic resection of superficial esophageal squamous cell carcinoma: a single institution experience. Gut Liver 2015; 9: 714-719

[19] Ishii N, Uchida S, Itoh T et al. Endoscopic submucosal dissection with a combination of small-caliber-tip transparent hood and flex knife for superficial esophageal neoplasia. Is it safe for elderly patients? Surg Endosc 2010; 24: 2110 - 2119

[20] Kawada K, Sugimoto T, Okada R et al. A case of simultaneous triple primary cancers of the hypopharynx, esophagus, and stomach which were dissected by endoscopic laryngo-pharyngeal surgery combined with endoscopic submucosal dissection. In. Open Journal of Gastroenterology 2018; 8: 94-102 